\title{
How sexual medicine is facing the outbreak of COVID-19: experience of Italian urological community and future perspectives
}

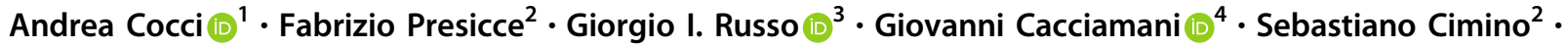 \\ Andrea Minervini ${ }^{1}$
}

Received: 18 March 2020 / Revised: 23 March 2020 / Accepted: 27 March 2020 / Published online: 14 April 2020

(c) The Author(s), under exclusive licence to Springer Nature Limited 2020

Since late December 2019, a novel coronavirus (COVID19) was identified as the cause of a cluster of pneumonia cases in the city of Wuhan, in the Hubei Province, China. It has rapidly spread throughout China, followed by an increasing number of cases world widely. The diffusion of coronavirus disease 2019 (COVID-19) has already reached the necessary epidemiological criteria for it to be declared pandemic, having infected more than 100,000 people in 100 countries.

While most people with COVID-19 show only mild or uncomplicated illness, 15-20\% experience severe disease that needs hospitalization and oxygen support, and 5-10\% require admission to an intensive care unit. In severe cases, COVID-19 can be complicated by an acute respiratory distress syndrome [1].

Italy is the nation outside China with the highest number of infected people and has been the first in Europe to face the outbreak of COVID-19. However, the available epidemiological data suggest that the Italian scenario may be repeated soon with a similar growth trend in the contagion throughout Europe and the United States (Fig. 1).

The exponential growth in the number of infected people experienced in Italy has represented a severe challenge for the national health system. The high number of patients who require hospitalization or in the worst case, access to

Giorgio I. Russo

giorgioivan1987@gmail.com

1 Department of Urology, Careggi Hospital, University of Florence, Florence, Italy

2 Department of Urology, Sapienza University, Rome, Italy

3 Urology section, Department of Surgery, University of Catania, Catania, Italy

4 USC Institute of Urology and Catherine and Joseph Aresty Department of Urology, University of Southern California, Los Angeles, CA, USA intensive care unit, makes us fear that the health system may have difficulty coping with it. Moreover from March 10, the Italian government has established draconian measures to limit the spread of the infection. These decisions clearly had a severe impact on everyday social and economic life, however, even within hospitals, daily activities have undergone major changes even in specializations, such as urology, that are not directly involved in the emergency.

Travel bans and restrictions caused the postponement or cancellation of several national/regional congresses. University lectures, oration courses, degrees ceremonies, and meetings have been canceled or scheduled through a conference call. In addition, to limit the spread of the infection and to increase the health resources to be allocated to the management of the epidemic, all the surgical procedures and nonurgent outpatient services for each specialty have been deferred $[2,3]$.

Furthermore, in some hospitals, the shortage of health personnel to manage the emergency has been so evident that it made it necessary to allocate even urologists to departments intended for the management and treatment of patients with COVID-19. Similarly in the worst scenarios, urological and other nonmedical specialization departments have been turned into medical departments due to the lack of beds.

Lastly, the high contagiousness of the virus combined with the lack of aids such as masks, filters, and hospital routes has subjected health workers to the risk of contagion with many infections recorded among health workers, causing further rearrangement within the healthcare professional.

All these considerations necessarily lead to rethinking how to organize our daily activities in a very different way than we used to until a few weeks ago. Unfortunately, probably all this will happen not only in Italy but also throughout Europe and for many months considering the epidemiological trends that are taking place. Indeed, several European meetings have been yet canceled and postponed. 
Fig. 1 Epidemic curve of confirmed COVID-19, by date of report and region through March 17, 2020. The graph shows the number of infected people by time in Italy, Spain, UK, USA and Germany from 23/01/2020 until 17/03/2020.

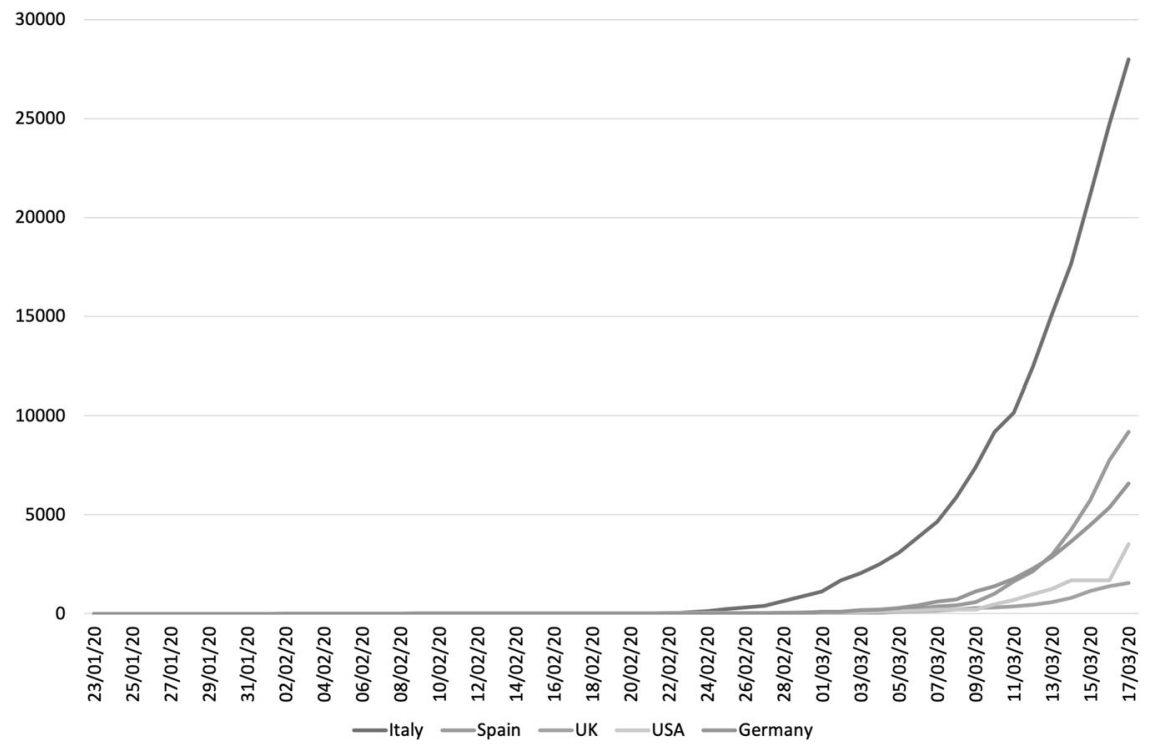

For the first time since the birth of the European Urology Society, the congress scheduled in Amsterdam on March 20th has been postponed to July.

Therefore, a coordinated response of urological community is desperately needed to prepare our systems to face this unprecedented challenge.

Surgical activity can be severely disrupted by an epidemic and a proper definition of which are the procedures that can be delayed is essential.

Many urological procedures may require admission in the Intensive Critical Units (ICUs) immediately after the surgery or due to major life-threating complications. In the Emergency that we are approaching, our urological community should make a conjoint effort in order to define properly the elective surgical procedure that can be postponed without compromising patient's prognosis and wellbeing. This important measurement for making available ICUs beds faces with a semantic issue: which surgeries can be defined as "strictly elective."

Together with all these conditions, also sexual medicine and others have to face with important changes in the clinical practice, taken into consideration the high prevalence of its interest in the general population $[4,5]$.

Outpatient activities will also have to be reorganized considering that some of the most important restrictive measures are social distancing and the limitation of travel. It will be necessary to establish which diagnostic procedures and which outpatient visits are to be considered mandatory and which instead are deferred. For example, it will be necessary to evaluate the risk/benefit of a procedure such as intravesical instillations that expose a patient, usually with high risk factors for COVID-19 (i.e., smoker status, chronic obstructive pulmonary disease) to repeated hospital access, a place statistically at high risk of contagion.
In these days, and probably this will be confirmed in the coming months, only emergency surgical procedures can be guaranteed, with considerable discomfort for all patients who fortunately have the clinical possibility to wait.

This will mean that patients with Peyronie's disease, requesting a penile implant, and anyone who needs treatment for erectile dysfunction will not be able to access treatment. We are not in a position to currently criticize these historical moments, but certainly, as health managers, we also have a duty to think about these patients.

Probably, and considering the devastating economic impact of the COVID-19 pandemic, these patients will be asked for a more substantial outlay to access their care, with a consequent imbalance between the economic classes. Finally, given the high risk of contagion for healthcare workers, the work organization within a department must also be re-modulated in order to minimize the risk of an epidemic spreading involving a large number of workers inside of a staff, effectively excluding an entire department for a few weeks at best.

All these critical issues are probably only the tip of the iceberg that the Italian urological community has had to face in daily on-field activities during the outbreak of COVID-19. Further difficulties are likely to emerge in the coming weeks as the outbreak continues.

Therefore, as a European/international urological community it is important to develop shared strategies to avoid the potential collateral damage of the COVID-19 pandemic for patients with urological diseases.

\section{Compliance with ethical standards}

Conflict of interest The authors declare that they have no conflict of interest. 
Publisher's note Springer Nature remains neutral with regard to jurisdictional claims in published maps and institutional affiliations.

\section{References}

1. Wu Z, McGoogan JM. Characteristics of and important lessons from the coronavirus disease 2019 (COVID-19) outbreak in China: summary of a report of 72314 cases from the Chinese center for disease control and prevention. JAMA. 2020. https://doi.org/10.1001/jama.2020.2648.

2. Chinazzi M, Davis JT, Ajelli M, Gioannini C, Litvinova M, Merler S, et al. The effect of travel restrictions on the spread of the
2019 novel coronavirus (COVID-19) outbreak. Science. 2020. https://doi.org/10.1126/science.aba9757.

3. Iacobucci G. Covid-19: GP surgeries close for two weeks after staff test positive. BMJ. 2020;368:m936.

4. Russo GI, di Mauro M, Cocci A, Cacciamani G, Cimino S, Serefoglu EC, et al. Consulting 'Dr Google' for sexual dysfunction: a contemporary worldwide trend analysis. Int J Impot Res. 2019. https://doi.org/10.1038/s41443-019-0203-2.

5. Cacciamani GE, Bassi S, Sebben M, Marcer A, Russo GI, Cocci A, et al. Consulting 'Dr. Google' for prostate cancer treatment options: a contemporary worldwide trend analysis. Eur Urol Oncol. 2019. https://doi.org/10.1016/j.euo.2019.07.002. 